\title{
Conserved meiotic mechanisms in the cnidarian Clytia hemisphaerica revealed by Spo11 knockout
}

Catriona Munro ${ }^{1,2}$, Hugo Cadis ${ }^{1,2}$, Evelyn Houliston ${ }^{2}$ and Jean-René Huynh ${ }^{1}$

${ }^{1}$ Center for Interdisciplinary Research in Biology, Collège de France, PSL Research University, CNRS UMR 7241 - INSERM U1050, 11 place Marcelin Berthelot, 75005, Paris, France

${ }^{2}$ Sorbonne Université, CNRS, Laboratoire de Biologie du Développement de

Villefranche-sur-mer (LBDV), 06230, France

\begin{abstract}
During meiosis, each duplicated chromosome pairs and recombines with its unique homolog to ensure the shuffling of genetic information across generations. Functional studies in classical model organisms have revealed a surprising diversity in the chronology and interdependency of the earliest meiotic steps such as chromosome movements, pairing, association via Synaptonemal Complex formation (synapsis), recombination and the formation of chiasmata. A key player is Spo11, an evolutionarily conserved topoisomerase-related transesterase that initiates meiotic recombination via the catalysis of programmed DNA double stranded breaks (DSBs). While DSBs are required for pairing and synapsis in budding yeast and mouse, alternative pathways are employed during female meiosis of the fruit fly and nematode Caenorhabditis elegans. Here, to provide a comparative perspective on meiotic regulation from a distinct animal clade, we chart gametogenesis in Clytia hemisphaerica jellyfish and examine the role of Spo11 using CRISPR-Cas9 mutants, generated clonally from F0 polyp colonies. Spo11 mutant females fail to assemble synaptonemal complexes and chiasmata, such that homologous chromosome pairs disperse during oocyte growth. Subsequent meiotic divisions are abnormal but produce viable progeny. Clytia thus shares an ancient eukaryotic dependence of synapsis and chromosome segregation on Spo11-generated DSBs. It provides a valuable additional experimental model for dissecting meiotic mechanisms during animal gametogenesis, and for building a comparative framework for distinguishing evolutionarily conserved versus flexible features of meiosis.
\end{abstract}

\section{Introduction}

Meiosis is a special type of cell division, whereby diploid germ cells undergo two rounds of nuclear division to produce haploid gametes. Homologous chromosomes (homologs) segregate during the first division (meiosis I) and chromatids separate during the second division (meiosis II). In order to segregate properly at meiosis I, each chromosome first needs to pair with its homolog during an extended Prophase $\mathrm{I}^{1,2}$. Pairing is sometimes initiated by a looser coupling or alignment of homologs. Pairing is then stabilized by the polymerisation of a proteinaceous structure called the synaptonemal complex (SC), which holds together homologous axes (synapsis) and promotes genetic recombination ${ }^{3}$. Exchanges of chromatids, or crossovers, allow for the formation of physical links, chiasmata, which maintain homologs associated in pairs upon depolymerization of the SC. Five stages have been defined during this extended Prophase I: 
leptotene is when homologs start to condense and become visible upon DNA staining; zygotene is when homologous chromosomes start to synapse; pachytene marks the completion of synapsis along the full length of every chromosome pair; at diplotene, the SC depolymerizes and homologs remain linked only by chiasmata; diakinesis is the final stage of prophase I, when chromosomes are condensed enough to make chiasmata detectable ${ }^{1,2}$. Individual meiotic processes and molecular machinery are well conserved among eukaryotes and likely descend from a common eukaryotic ancestor ${ }^{4}$, however, the interdependencies between them vary between species.

This paradox has been highlighted by studies of Spo11, a meiosis-specific enzyme highly conserved across eukaryotes that is required for the formation of double-stranded breaks (DSBs) and the initiation of meiotic recombination ${ }^{5-14}$. In many of the species studied, Spo11-generated DSBs are required for pairing and synapsis of the replicated homologous chromosomes, with crossover sites (chiasmata) required subsequently for maintaining the pairs until their correct segregation during meiotic divisions 5,8-11,13,14. There are, however, several exceptions, notably in the classical animal model species Caenorhabditis elegans and female Drosophila melanogaster ${ }^{6,7}$. When the Spo11 gene is mutated/knocked down in these species, recombination is lost and meiotic segregation is aberrant, however pairing and synapsis are unaffected (wild-type D. melanogaster males in any case do not undergo meiotic recombination). All the animal models in which Spo11 function has been tested so far belong to the clade Bilateria (Figure 1A). A valuable evolutionary perspective on meiotic processes could be provided from its sister clade Cnidaria, which has diversified in parallel to give rise to a wide range of species including corals, anemones, and jellyfish. The jellyfish Clytia hemisphaerica is now established as a cnidarian model species well suited for experimental manipulation, with the simple organization and transparency of the gonads making them particularly attractive material for analyzing gametogenesis regulation in vivo ${ }^{15,16}$. Furthermore, particularities of its life cycle (Figure 1B) conveniently enable phenotypes of gene mutations targeted by CRISPR-Cas9 to be studied in clonally-produced F0 generation jellyfish, which bud from stable, vegetatively-propagating polyp colonies ${ }^{17}$.

\section{Results and Discussion}

\section{Mapping early meiosis in Clytia hemisphaerica}

To establish a framework for studies of early meiotic mechanisms in Clytia, we first documented the formation of the gonads in male and female jellyfish, which are released continuously from the polyp colony. In newly released jellyfish, four small patches of germ-line precursor cells enriched in Piwi protein can be distinguished midway along each radial canal at the sites of the future gonads (Figure 1C, 1D, Supplementary Figure S1A,B). The gonads develop and expand as the jellyfish grows to sexual maturity, from about $1 \mathrm{~mm}$ to $1 \mathrm{~cm}$ in diameter over $2-3$ weeks, remaining clearly visible within the transparent animal. They maintain a simple overall organization as the germline cells proliferate and differentiate between two somatic tissue layers: gastrodermis and epidermis (Figure 1C). In females, germ cells entering meiosis can be detected by 4-5 days, when oocytes of all stages of prophase I can be identified (Figure 1C, 1E). Hoechst staining revealed classic figures of condensed chromatin during 
leptotene-zygotene, and structured parallel tracks indicative of pairing and synapsis in pachytene (Figure 1E). Diplotene nuclei show a distinctive Hoechst-negative "hole" within which one or more diffuse Hoecht-positive patches appear as the oocyte grows (Figure 1E). Telomere FISH revealed characteristic "bouquet" configurations in leptotene/zygotene oocytes (Figure 1E). Diplotene arrested oocytes undergo extensive growth, reaching 180-200 $\mu \mathrm{m}$ in diameter. Fully grown oocytes become competent to undergo meiotic maturation and spawning, a process triggered daily in mature ovaries by a peptide hormone $(\mathrm{MIH})$ secreted in response to light ${ }^{18-20}$. Following each spawning, "stage I" growing oocytes of about $50 \mu \mathrm{m}$ in diameter grow to full size in around 13-15 hours ${ }^{16,20}$. At the end of this growth phase the chromosomes recondense in preparation for diakinesis ${ }^{16}$. Clytia male jellyfish undergo a longer period of proliferation of germ-line precursors than females (Figure 1C, Supplementary Figure S1C,D). After 2 weeks, prophase I spermatogonia are distributed in layers, with leptotene/zygotene cells closest to the gastrodermis, then pachytene, spermatids, and finally spermatozoa closest to the epidermis (Figure 1C, Supplementary Figure S1C,D). Daily sperm release occurs approximately $1.5 \mathrm{~h}$ after light exposure. Within each subsequent 24 hour period, cohorts of spermatogonia enter and progress through pachytene, while in parallel late pachytene cells enter diplotene and progress through the two meiotic divisions.

\section{Synapsis in Clytia is Spo11 dependent}

We identified a single conserved Clytia Spo11 gene using genome and transcriptome data ${ }^{21}$, along with conserved homologs of other key meiosis genes, coding for synaptonemal complex components Sycp1 and Sycp3, and repair pathway proteins Rad51, Mlh1, Dmc1 (Supplementary Figure S2, Supplementary trees and alignments). We mutated the Clytia Spo11 gene by injection into eggs of single CRISPR-Cas9 guide RNAs targeting either exon 1 or exon 2 (Figure 2A, Supplementary Figure S3). This generated F0 male and female Spo11 mutant polyp colonies that each contained small sets of insertion and deletion events around the targeted site, but no detectable wild-type Spo11 sequence (Table 1, Supplementary Figure S3). This typical outcome of CRISPR-Cas9 mutagenesis in Clytia results from very active but inaccurate Microhomology Mediated End-Joining (MMEJ) repair of DNA double strand breaks in the embryo, followed by reduction in genotypic complexity during larval metamorphosis ${ }^{17}$. Mutant alleles were consistent among jellyfish produced from the same mutant polyp colony.

Pachytene is characterized by a fully formed synaptonemal complex (SC), which mediates tight association between each pair of duplicated chromosomes ${ }^{3}$. We visualized the SC during pachytene in both males and females using antibodies generated to Clytia Sycp1, which forms the transverse filaments of the SC, and Sycp3, a component of the axial elements associated with each individual chromatid pair (Figure 2B). In Spo11 mutants we detected morphologically distinct pachytene-like stages within both male and female gonads but SCs were absent, anti-Scyp1 decorating only occasional short chromosome stretches (Figure 2B, C). Consistently, ladder-like synaptonemal complexes could be detected by electron microscopy in wild-type pachytene oocytes, (Figure 2D), but not (or very rarely for some strains) in Spo11 mutant pachytene oocytes (Figure 2E; Supplementary Table S1). We performed telomere FISH for two female Spo11 mutants and could distinguish telomere bouquets characterizing 
leptotene/zygotene (Supplementary Figure S4), suggesting that interchromosomal interactions during these early stages are dependent neither on Spo11 function nor on synapsis. The first observable defect in Clytia Spo11 mutants is thus a failure in synapsis, i.e. in the correct association of paired chromosomes via SC formation.

\section{Chiasmata-mediated chromosome segregation fails in Spo11 mutants}

A convenient feature of Clytia oogenesis for analyzing meiotic recombination events is that paired chromosomes connected at chiasmata sites can be readily observed towards the end of the oocyte growth phase, as the duplicated diplotene chromosomes recondense ahead of diakinesis. Within the enlarged oocyte nucleus ('Germinal Vesicle') of wild type oocytes, 15 pairs of chromosomes with 1-2 crossovers per pair are clearly distinguishable (Figure 3A top panel). In all female Spo11 mutants, however, we consistently distinguished 30 scattered univalents at this stage (Figure 3A bottom panel), with only occasional hints of connections between them. This phenotype strongly suggests that, as in many species, Spo11-mediated DNA double strand breaks are necessary for the formation of chiasmata, which in turn are required to maintain chromosome pairing during oocyte growth. We confirmed the absence of crossover events in Spo11 mutant females at early diplotene stages using an antibody raised to Clytia Mlh1 (Supplementary trees and alignments). Mlh1 localizes to and stabilizes meiotic crossovers across many species ${ }^{22,23}$. In wildtype diplotene cells, clear Mlh1 foci were observed (Figure 3B top panel), with a mean of $32.75 \pm 16.38$ foci per nucleus $(n=21$; raw counts in supplementary file 1). In contrast, diplotene nuclei in Spo11 mutants completely lacked Mlh1 staining (Figure 3B bottom panel), in line with the observed loss of chiasma in fully grown oocytes.

The loss of chiasma and resultant scattering of individual duplicated chromosomes in fully grown oocytes severely impacts the subsequent meiotic divisions. When Spo11 mutant oocytes are induced by $\mathrm{MIH}$ treatment to undergo meiotic maturation, univalents fail to align on a metaphase plate at the first division but remain scattered across defective spindle structures (Figure 4A,C). The observed chromosome configurations indicated that polar body formation in Spo11 mutant oocytes frequently aborted during anaphase I (Figure 4B, D). Despite these severe abnormalities in the meiotic divisions, resulting typically in retention of two or four haploid sets of chromosomes in the oocyte, Spo11 mutant oocytes could be successfully fertilized with wildtype sperm at near wildtype frequencies. In most cases the resultant zygotes developed into planula larvae and could undergo metamorphosis to the polyp stage (Supplementary Table S2). Similarly, Spo11 mutant males show no loss of fertility, producing functional sperm capable of fertilizing wildtype oocytes to generate metamorphosis-competent larvae (Supplementary Table S2).

In this study we show that meiosis in Clytia hemisphaerica relies on classical mechanisms of homologous chromosome pairing, synapsis, and recombination, as summarized in Figure 4E. The sequence of events is particularly accessible for observation in female jellyfish, where germ cells are larger, diplotene is prolonged during oocyte growth, and the meiotic divisions can conveniently be triggered using synthetic $\mathrm{MIH}$. During leptotene/zygotene, clustering of telomeres indicates active interchromosomal interactions. During pachytene, chromosomes 
become closely associated during synapsis as the SC is fully established. In diplotene, we see the emergence of stable crossovers, detected first as Mlh1 foci and then later in the fully grown oocytes as attachment points between homologous chromosome pairs. Spo11 function thus appears not to impact the initial progression of events following meiotic entry in male and female Clytia germ cells, but is essential for correct formation of the synaptonemal complex. Due to interdependencies of the subsequent events, establishment of crossovers, their tethering of chromosome pairs during oocyte growth, and proper segregation of chromosomes on the first meiotic spindle also require Spo11 function ${ }^{11}$. Severe defects in chromosome segregation during the meiotic divisions upon oocyte maturation result in defective polar body emission and thus increased oocyte ploidy, but Spo11 mutant gametes can nevertheless fertilize/be fertilized. Furthermore the hybrid zygotes develop into metamorphosis-competent larvae, implying mechanisms during embryogenesis to handle abnormal ploidy, which will be exciting to address in future studies.

The sequence of meiotic events and their interdependencies revealed by Spo11 knockout in Clytia closely match ones well described across eukaryotic model organisms including mice, zebrafish, budding yeast, filamentous fungi and plants including Arabidopsis thaliana ${ }^{1}$. They are thus likely governed by ancestral and conserved mechanisms. Not all animals, however, share the same relationships between Spo11 function and subsequent events. Alternative modes of DSB-independent synapsis and pairing have notably been described in the traditional animal model species $C$. elegans and $D$. melanogaster ${ }^{6,7}$. In $D$. melanogaster, male chromosomes show no recombination, while chromosome pairing in females initiates prior to meiotic entry in proliferating germ cells ${ }^{24,25}$. This "pre-pairing" may represent a specialization to facilitate later SC formation within the particular context of the developing Drosophila germ cells. In $C$. elegans, recombination-independent chromosome pairing occurs at "pairing centers", specialized sites at chromosome ends, which also promote initiation of synapsis ${ }^{26}$. The involvement of pairing centers in promoting synapsis likely evolved within the Caenorhabditis clade, since a classic role for Spo11 in chromosome pairing, synapsis, and crossover formation has been uncovered in another nematode species, Pristionchus pacificus ${ }^{14}$. In the planarian Schmidtea mediterranea, pairing and synapsis in males are not dependent on Spo11, and it is proposed that the telomere cluster plays a role in initiating SC formation, which in turn drives homolog pairing ${ }^{12}$. SC nucleation mechanisms are as yet unknown in this species ${ }^{12}$.

To conclude, this work establishes the jellyfish Clytia hemisphaerica as an excellent complementary model for addressing early meiotic mechanisms based on mechanisms largely conserved from a distant eukaryotic ancestor. Core synaptonemal complex proteins from cnidarians show high sequence similarity with most other animals, contrasting with marked diversification in the ecdysozoan clade (including D. melanogaster and C. elegans) ${ }^{27}$. Clytia gonads are transparent, simply organized, and accessible to manipulation and observation within the animal. Gametes are generated continuously with cycles of oocyte growth and spermatogenesis linked to light-triggered daily spawning, enabling precise timed access to particular meiotic stages; CRISPR/Cas9 knockout is highly efficient and phenotypes can be examined directly in jellyfish from F0 mutant polyp colonies. The short cycles of gametogenesis favor live imaging approaches offering exciting perspectives to exploit recent technical advances 
to produce transgenic Clytia expressing tagged proteins ${ }^{28}$. We anticipate that Clytia will provide new insights into conserved meiotic mechanisms as well as a valuable evolutionary comparative perspective to our understanding of diversity in meiotic mechanisms across animals.

\section{Methods}

\section{Clytia hemisphaerica cultures and maintenance}

Clytia hemisphaerica cultures were maintained as described previously ${ }^{29}$. Several wildtype strains were used in this study, with consistent results, including female strains $Z 11, Z 4 B, A 2$, Z21; Z28; and male strains Z13, Z23, A3. CRISPR-Cas9 mutant jellyfish were generated by injecting Z11 oocytes and fertilizing them with Z13 sperm.

\section{Gene identification and homology}

Clytia hemisphaerica genes, for antibody, guide RNA, and FISH probe design, were identified via BLAST against the Clytia genome http://marimba.obs-vlfr.fr/ ${ }^{21}$. Gene alignments were made using MAFFT v7.453 L-INS-i ${ }^{30}$, and gene phylogenies generated with IQ-TREE ${ }^{31}$ using a $\mathrm{LG}+\mathrm{F}+\mathrm{R} 4$ model. Gene trees and alignments are available as supplementary data (folder containing phylogenetic trees, alignments, and amino acid sequences).

\section{Spo11 CRISPR-Cas9 knockouts}

Spo11 mutants were generated as described previously ${ }^{17,18}$. Candidate guide RNAs with predicted cut sites between microhomologies were identified using http://www.rgenome.net/mich-calculator/, and off-target sites using http://crispor.tefor.net/. crRNA sequences are listed in table S3. Spo11 small guide RNA (sgRNA) was generated by hybridizing $200 \mu \mathrm{M}$ crRNA and $200 \mu \mathrm{M}$ tracrRNA in the presence of 1x Hybridization buffer (Integrated DNA Technologies, Coralville, IA), $95^{\circ} \mathrm{C}$ for $5 \mathrm{~min}$, cooled to $25^{\circ} \mathrm{C}$ at $-0.1^{\circ} \mathrm{C} / \mathrm{sec}$. Two separate sgRNAs were injected, targeting regions of exon 1 and of exon 2 . Prior to injection, $0.5 \mu \mathrm{l}$ of hybridized crRNA/tracrRNA $(60 \mu \mathrm{M})$ was mixed with $2 \mu$ l Cas9 protein $(10 \mu \mathrm{M})$, incubated at room temperature for 10 minutes, adjusted with $0.84 \mu \mathrm{l}$ Cas 9 Buffer $(10 \mathrm{mM}$ Hepes, $350 \mathrm{mM} \mathrm{KCl}$ ), and centrifuged at $14,000 \mathrm{rpm}$ for 10 minutes at $12^{\circ} \mathrm{C}$. Injection, fertilization and subsequent metamorphosis of larvae was conducted as described previously ${ }^{17,18}$.

\section{Genotyping}

Genomic DNA was extracted from a single jellyfish using DNeasy blood/tissue extraction kit (Qiagen). DNA around the target site was amplified using Phusion DNA polymerase (NEB, Ipswich, MA). PCR primer sequences are listed in table S3. PCR products were cleaned up and sequenced. Genotypes of Clytia mutant strains are in Table 1 and Supplementary Figure S3. 


\section{Antibodies}

Antibodies recognizing Clytia Sycp1 were generated in rabbits using two peptides IRNWKSEKEMELKMKD-Cys (75-90aa) and Cys-PKAMTPKTPNMRYS (833-846aa). Antibodies recognizing Clytia Sycp3 were generated in rats using two peptides ENAPAEEAPAISGK-Cys (25-38aa) and GRKRPAPHISHT-Cys (39-50aa). Antibodies recognising Clytia Mlh1 and CenH3-1 were generated in mice using a recombinant protein generated in $E$. coli against the full length optimized sequence. Antibodies recognising Clytia Piwi1 were raised in rabbits using a recombinant protein generated in $E$. coli against

SGEPVQILTNYFKVDKMPRFEGLHQYVVAFDPDIQSQKLKGFLLFSMQDVIGEVKVFDGMSLFL PRKLAEPVVERCVETRDGSSIKVKITHTNEVPVNSPQVVQLM (115-220aa) fused with an N-his Tag. All antibodies were generated and affinity-purified by Proteogenix (Schiltigheim, France).

For immunostaining, anti-Sycp1 antibodies were diluted 1:2000 for sycp1\#4 and 1:1000 for sycp1\#2. Sycp3\#2 was diluted 1:400. Mlh1\#2 was diluted 1:250. Piwi\#1 and Piwi\#2 were diluted 1:2000. Secondary antibodies (anti-mouse Mlh1; anti-rabbit Sycp1, Piwi) were diluted 1:200. Rat monoclonal anti tyrosinated tubulin YL1/2 was diluted 1:500 (Thermo Fisher Scientific).

\section{Immunostaining}

For anti-Sycp1, anti-Sycp3 and anti-Mlh1 staining, samples (whole 1 week jellyfish or dissected gonads) were fixed in $1 \%$ formaldehyde in methanol at $-20^{\circ} \mathrm{C}$ for at least 2 hours (up to overnight). Methanol fixed samples are left to warm gradually to room temperature ( $\sim 30$ mins) before rehydration to PBS. For anti-tubulin and anti-Piwi1 staining, samples were fixed for $2 \mathrm{~h}$ at room temperature in IF fix (Hepes pH 6.9, 0.1M, EGTA pH 7.250 mM, MgSO4 10mM, Maltose $80 \mathrm{mM}$, Triton $100 \times 0.2 \%, 4 \%$ paraformaldehyde) for 2 hours before proceeding to washes and staining.

All antibody staining then proceeded with the following washes: PBS-Triton $0.01 \% 3 \times 10$ mins, PBS-Triton $0.2 \% 40$ mins, PBS-Triton 0.01\% 2 x 10 mins, PBS/BSA 3\% 20 mins-1h. Primary antibodies were diluted with PBS/BSA $3 \%$ and incubated with the sample at $4{ }^{\circ} \mathrm{C}$ overnight. The next day, wash PBS-Triton $0.01 \% 3 \times 10$ mins. Dilute secondary antibody in PBS-Triton $0.01 \%$ and Hoechst $333421: 5000$, incubate with sample $2 \mathrm{~h}$ at room temperature or overnight $4^{\circ} \mathrm{C}$. Wash in PBS-Triton 0.01\% 4 × 5 min, mount in Citifluor antifade mountant (Citifluor-EMS).

\section{Telomere FISH}

Telomere FISH was conducted using $\mathrm{Cy} 3$ and Alexa647-labeled G-Rich telomere probe (Eurogentec, PN-TG020-005, PN-TG050-005) targeting repeats of TTAGGG. Probes were resuspended in formamide at $50 \mu \mathrm{M}$. A working aliquot was stored at $4^{\circ} \mathrm{C}$ for regular use, remaining aliquots were stored at -20 for longer-term storage. Hybridization buffer solution was made fresh each time before starting the FISH protocol: $20 \mathrm{mM}$ Sodium phosphate $\mathrm{Na}_{2} \mathrm{HPO}_{4} \mathrm{pH}$ 7.4, 20mM Tris- $\mathrm{HCl}$ pH 7.4, 60\% Deionized Formamide, 2xSSC, tRNA 1x, Heparin 1x. 
FISH was conducted as follows: samples (up to 1 week jellyfish (5-8 per tube), isolated oocytes) were fixed overnight at room temperature in HEM fix (Hepes pH 6.9, 0.1M, EGTA pH 7.250 $\mathrm{mM}$, MgSO4 10mM, 4\% formaldehyde), washed 3x 10 mins with PBS-Tween $0.1 \%$, and then dehydrated and rehydrated in methanol on ice (PBST/methanol 50\% 1x 10 min, Methanol 100\% $2 \times 10 \mathrm{~min}, \mathrm{PBST} / \mathrm{methanol} 50 \% 1 \times 10 \mathrm{~min}$ ). Samples were then washed in $2 \times \mathrm{SSC}-0.1 \%$ Tween $(\mathrm{pH} 7), 3 \times 5 \mathrm{~min}$. They were incubated in RNaseA solution $(100 \mathrm{mg} / \mathrm{ml})$ in an oven at $37^{\circ} \mathrm{C}$ for $1 \mathrm{~h}$. Then washed $3 \times 5$ mins in $2 x S S C-0.1 \%$ Tween $(\mathrm{pH} 7)$ at room temperature. Then transferred to $50 \%$ hybridization buffer $/ 2 \times S S C$ at $80^{\circ} \mathrm{C}$ for 5 mins in a water bath. In parallel, the probe $\left(1 \mu \mathrm{l}\right.$ in $99 \mu \mathrm{l}$ hybridization buffer) was heated $90^{\circ} \mathrm{C}$ for 5 mins. As quickly as possible, we removed as much $50 \%$ hybridization buffer/2xSSC as possible and added the probe to the sample, gently flicking the tube to mix. The sample was then incubated 10 mins at $85^{\circ} \mathrm{C}$ in a water bath. Subsequently, samples were placed in the dark at room temperature for 1 hour for hybridization. After hybridization, samples were washed 2x 10 mins with 2xSSC- $0.1 \%$ Tween at $60^{\circ} \mathrm{C}$ in an oven (2xSSC- $0.1 \%$ was preheated to $60^{\circ} \mathrm{C}$ prior to washing). Samples were washed once with $2 x S S C-0.1 \%$ Tween at room temperature, then stained with Hoechst dye 33342 1:5000 (30 mins - 1h), washed 3x5 mins with 2xSSC, and mounted in Citifluor.

\section{In-situ hybridization}

In-situ hybridization probes were produced as described previously ${ }^{32}$, and in-situs performed following the urea-based protocol ${ }^{33}$.

\section{Transmission Electron Microscopy (TEM)}

For TEM, gonads were dissected from 4 and 5 day old female jellyfish in $400 \mu \mathrm{M}$ menthol in seawater (Sigma-Aldrich, \#M2772, diluted from a 1M stock solution in ethanol). Fixation and embedding in Spurr resin were performed as described previously ${ }^{34}$.

\section{Fertilization Experiments}

Embryos were generated as described previously ${ }^{29}$ with the following crosses: mutant female $x$ wildtype male, mutant male $x$ wildtype female, with a control of wildtype female $x$ wildtype male. Mutant or wildtype eggs were collected and added to glass dishes with the same volume of liquid. For female mutant crosses, the same volume of wildtype sperm was added to mutants as to wildtype. If wildtype fertilization rate was low or development disrupted, indicative of too much or too little sperm, results were discarded. In all experiments, adequate sperm concentration was verified by the presence of $\sim 12$ sperm around each egg. Raw counts are available in supplementary file 2 .

\section{Meiotic maturation}

Meiotic maturation was induced as described previously ${ }^{18}$. Briefly, gonads were dissected from mutant and wildtype female jellyfish adapted to an afternoon light cycle, and were maintained 
overnight in dishes with the light on. The following morning, fully grown oocytes were isolated, and the MIH peptide (WPRPamide) was added to seawater containing the oocytes for a final concentration of $100 \mathrm{nM}$. Oocytes were fixed every 5 minutes, starting 25 minutes after addition of MIH.

\section{Acknowledgements}

The authors declare that they have no competing interests. We thank Tsuyoshi Momose for advice and help with CRISPR/Cas9 protocols and culture of mutant colonies; Lisa Rouressol for assisting with exploration of male Clytia gametogenesis; Sophie Pagnotta (Plateforme Commune de Microscopie Electronique, Université Côte d'Azur) for Transmission Electron Microscopy; Régis Lasbleiz and Axel Duchene at the Marine Resources Centre (CRBM) and Sébastien Schaub at the imaging platform (PIV) of Institut de la Mer de Villefranche. CRBM and PIV are supported by EMBRC-France, whose French state funds are managed by the ANR within the Investments of the Future program under reference ANR-10-INBS-02. This project received funding from the European Union's Horizon 2020 research and innovation programme under the Marie Skłodowska-Curie grant agreement No 841433 (JOLI), from the CNRS-INSB "Diversity of Biological Mechanisms" program, and from the European Union's Horizon 2020 research and innovation programme No 730984, ASSEMBLE Plus project, JRA3. The J.-R.H. lab is supported by CNRS, INSERM, Collège de France, La Fondation pour la Recherche Médicale (FRM) (Equipes FRM DEQ20160334884), Agence Nationale de la Recherche (ANR) (ANR-15-CE13-0001-01, AbsCyStem) and the Bettencourt Schueller Foundation.

\section{References}

1. Zickler, D. \& Kleckner, N. Recombination, Pairing, and Synapsis of Homologs during Meiosis. Cold Spring Harb. Perspect. Biol. 7, a016626 (2015).

2. Zickler, D. \& Kleckner, N. A few of our favorite things: Pairing, the bouquet, crossover interference and evolution of meiosis. Semin. Cell Dev. Biol. 54, 135-148 (2016).

3. Page, S. L. \& Hawley, R. S. The Genetics and Molecular Biology of the Synaptonemal Complex. Annu. Rev. Cell Dev. Biol. 20, 525-558 (2004).

4. Goodenough, U. \& Heitman, J. Origins of Eukaryotic Sexual Reproduction. Cold Spring Harb. Perspect. Biol. 6, a016154 (2014).

5. Keeney, S., Giroux, C. N. \& Kleckner, N. Meiosis-specific DNA double-strand breaks are catalyzed by Spo11, a member of a widely conserved protein family. Cell 88, 375-384 (1997).

6. McKim, K. S. et al. Meiotic Synapsis in the Absence of Recombination. Science 279, 876-878 (1998).

7. Dernburg, A. F. et al. Meiotic Recombination in C. elegans Initiates by a Conserved Mechanism and Is Dispensable for Homologous Chromosome Synapsis. Cell 94, 387-398 (1998).

8. Romanienko, P. J. \& Camerini-Otero, R. D. The Mouse Spo11 Gene Is Required for Meiotic Chromosome Synapsis. Mol. Cell 6, 975-987 (2000).

9. Baudat, F., Manova, K., Yuen, J. P., Jasin, M. \& Keeney, S. Chromosome Synapsis Defects and Sexually Dimorphic Meiotic Progression in Mice Lacking Spo11. Mol. Cell 6, 989-998 
(2000).

10. Grelon, M., Vezon, D., Gendrot, G. \& Pelletier, G. AtSPO11-1 is necessary for efficient meiotic recombination in plants. EMBO J. 20, 589-600 (2001).

11. Storlazzi, A. et al. Meiotic double-strand breaks at the interface of chromosome movement, chromosome remodeling, and reductional division. Genes Dev. 17, 2675-2687 (2003).

12. Xiang, Y., Miller, D. E., Ross, E. J., Sánchez Alvarado, A. \& Hawley, R. S. Synaptonemal complex extension from clustered telomeres mediates full-length chromosome pairing in Schmidtea mediterranea. Proc. Natl. Acad. Sci. 111, E5159-E5168 (2014).

13. Blokhina, Y. P., Nguyen, A. D., Draper, B. W. \& Burgess, S. M. The telomere bouquet is a hub where meiotic double-strand breaks, synapsis, and stable homolog juxtaposition are coordinated in the zebrafish, Danio rerio. PLOS Genet. 15, e1007730 (2019).

14. Rillo-Bohn, R. et al. Analysis of meiosis in Pristionchus pacificus reveals plasticity in homolog pairing and synapsis in the nematode lineage. eLife 10, e70990 (2021).

15. Houliston, E., Momose, T. \& Manuel, M. Clytia hemisphaerica: a jellyfish cousin joins the laboratory. Trends Genet. 26, 159-167 (2010).

16. Jessus, C., Munro, C. \& Houliston, E. Managing the Oocyte Meiotic Arrest-Lessons from Frogs and Jellyfish. Cells 9, 1150 (2020).

17. Momose, T. et al. High doses of CRISPR/Cas9 ribonucleoprotein efficiently induce gene knockout with low mosaicism in the hydrozoan Clytia hemisphaerica through microhomology-mediated deletion. Sci. Rep. 8, 11734 (2018).

18. Quiroga Artigas, G. et al. A G protein-coupled receptor mediates neuropeptide-induced oocyte maturation in the jellyfish Clytia. PLOS Biol. 18, e3000614 (2020).

19. Quiroga Artigas, G. et al. A gonad-expressed opsin mediates light-induced spawning in the jellyfish Clytia. eLife 7, e29555 (2018).

20. Amiel, A. \& Houliston, E. Three distinct RNA localization mechanisms contribute to oocyte polarity establishment in the cnidarian Clytia hemisphærica. Dev. Biol. 327, 191-203 (2009).

21. Leclère, L. et al. The genome of the jellyfish Clytia hemisphaerica and the evolution of the cnidarian life-cycle. Nat. Ecol. Evol. 3, 801-810 (2019).

22. Baker, S. M. et al. Involvement of mouse Mlh1 in DNA mismatch repair and meiotic crossing over. Nat. Genet. 13, 336-342 (1996).

23. Hunter, N. \& Borts, R. H. Mlh1 is unique among mismatch repair proteins in its ability to promote crossing-over during meiosis. Genes Dev. 11, 1573-1582 (1997).

24. Christophorou, N., Rubin, T. \& Huynh, J.-R. Synaptonemal Complex Components Promote Centromere Pairing in Pre-meiotic Germ Cells. PLOS Genet. 9, e1004012 (2013).

25. Christophorou, N. et al. Microtubule-driven nuclear rotations promote meiotic chromosome dynamics. Nat. Cell Biol. 17, 1388-1400 (2015).

26. MacQueen, A. J. et al. Chromosome Sites Play Dual Roles to Establish Homologous Synapsis during Meiosis in C. elegans. Cell 123, 1037-1050 (2005).

27. Fraune, J. et al. Hydra meiosis reveals unexpected conservation of structural synaptonemal complex proteins across metazoans. Proc. Natl. Acad. Sci. 109, 16588-16593 (2012).

28. Weissbourd, B. et al. A genetically tractable jellyfish model for systems and evolutionary 
neuroscience. Cell 184, 5854-5868.e20 (2021).

29. Lechable, M. et al. An improved whole life cycle culture protocol for the hydrozoan genetic model Clytia hemisphaerica. Biol. Open 9, (2020).

30. Katoh, K. \& Standley, D. M. MAFFT Multiple Sequence Alignment Software Version 7: Improvements in Performance and Usability. Mol. Biol. Evol. 30, 772-780 (2013).

31. Minh, B. Q. et al. IQ-TREE 2: New Models and Efficient Methods for Phylogenetic Inference in the Genomic Era. Mol. Biol. Evol. 37, 1530-1534 (2020).

32. Lapébie, P. et al. Differential Responses to Wnt and PCP Disruption Predict Expression and Developmental Function of Conserved and Novel Genes in a Cnidarian. PLOS Genet. 10, e1004590 (2014).

33. Sinigaglia, C., Thiel, D., Hejnol, A., Houliston, E. \& Leclère, L. A safer, urea-based in situ hybridization method improves detection of gene expression in diverse animal species. Dev. Biol. 434, 15-23 (2018).

34. Eisenman, E. A. \& Alfert, M. A new fixation procedure for preserving the ultrastructure of marine invertebrate tissues. J. Microsc. 125, 117-120 (1982). 
Figures and Tables

Table 1. Summary of genotypes and phenotypes of Spo11 mutant colonies in this study

\begin{tabular}{|l|l|l|l|l|l|l|}
\hline $\begin{array}{l}\text { Colony } \\
\text { name }\end{array}$ & Sex & $\begin{array}{l}\text { Exon } \\
\text { targeted }\end{array}$ & $\begin{array}{l}\text { Most common } \\
\text { alleles }\end{array}$ & $\begin{array}{l}\text { Synaptonemal } \\
\text { complex } \\
\text { (Scyp1) }\end{array}$ & $\begin{array}{l}\text { Chiasma } \\
\text { in fully } \\
\text { grown } \\
\text { oocytes }\end{array}$ & Fertility \\
\hline Q9 & Female & 1 & $\begin{array}{l}\text { 5bp deletion; } 6 \mathrm{bp} \\
\text { insertion }\end{array}$ & Absent & No & Fertile \\
\hline Q5 & Female & 1 & $\begin{array}{l}\text { 6bp insertion; } 5 \mathrm{bp} \\
\text { insertion; } 5 \mathrm{bp} \\
\text { deletion; } 2 \mathrm{bp} \\
\text { insertion }\end{array}$ & Absent* & No & Fertile \\
\hline P16 & Female & 2 & $\begin{array}{l}\text { 10bp deletion; } \\
\text { 184bp deletion; } \\
\text { 5bp deletion/3bp } \\
\text { substitution }\end{array}$ & Absent & No & Fertile \\
\hline P11 & Male & 1 & $\begin{array}{l}\text { 1bp deletion; large } \\
\text { deletion/insertion }\end{array}$ & Absent & N/A & Fertile \\
\hline
\end{tabular}

* Occasional partial SCs detected 


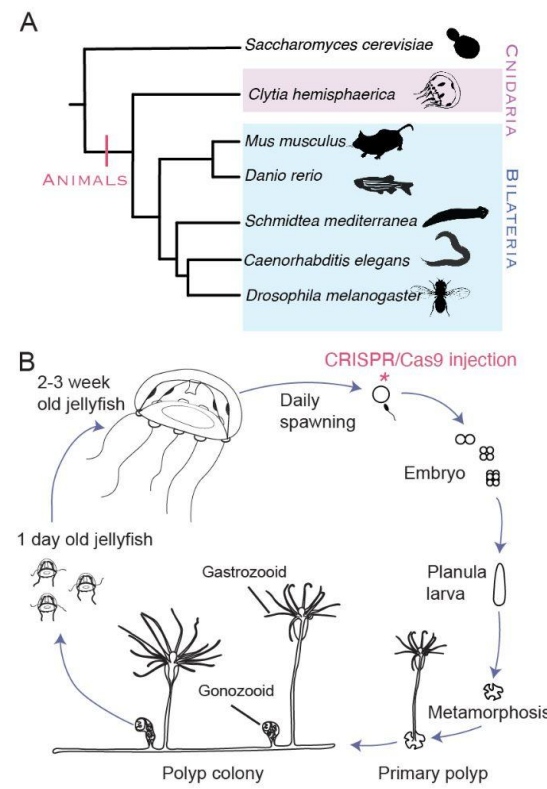

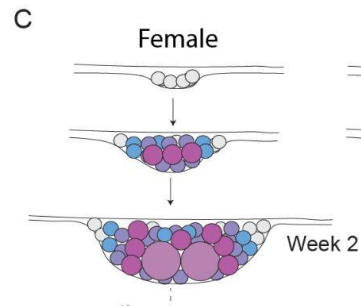

cross-section

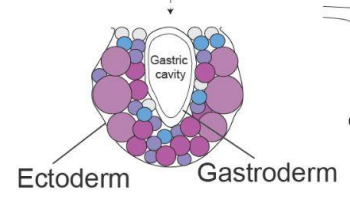

Ectoderm

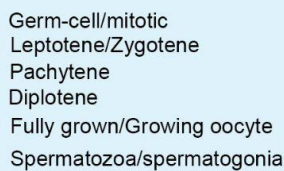

Spermatozoa/spermatogonia
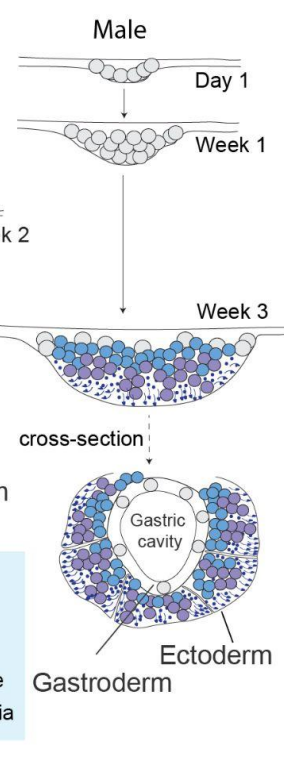
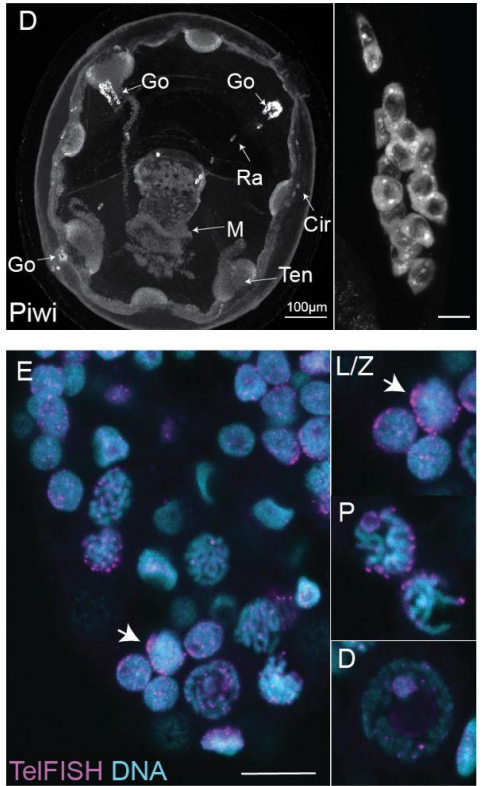

Figure 1. Phylogenetic placement, life-cycle, and gonad organization of Clytia hemisphaerica. A. Evolutionary relationships among major animal meiosis models. B. Life-cycle of Clytia hemisphaerica. The polyp colony propagates asexually for many years in the lab. Specialized polyps called gonozooids release jellyfish continuously. Sexually mature jellyfish release eggs or sperm daily in response to dark-light transitions. The embryo develops into a "planula" larva which settles and metamorphoses to form a primary polyp. To achieve gene knockouts, CRISPR/Cas9 is injected in the mature egg immediately prior to fertilization. C. Development and organization of the male and female gonad from the first day of jellyfish release to sexual maturity. D. Maximum intensity projection of a confocal image stack of a three day old jellyfish stained with anti-Piwi. Go = gonad, $\mathrm{Ra}=$ radial canal, $\mathrm{M}=$ manubrium $/ \mathrm{mouth}$, Ten = tentacle bulb, Cir = circular canal. Scale is $100 \mu \mathrm{m}$. Similar projection from a 3 day old gonad is shown on the right, scale is $10 \mu \mathrm{m}$. E. Confocal section showing oocytes at mixed meiotic stages within the gonad of a one week female jellyfish stained with telomere FISH (magenta) and Hoechst (cyan). Inset shows close-up of representative nuclei at different prophase I stages, L/Z = leptotene/zygotene, $\mathrm{P}=$ pachytene, $\mathrm{D}=$ diplotene. Arrow indicates telomere bouquet. Scale is $10 \mu \mathrm{m}$. 

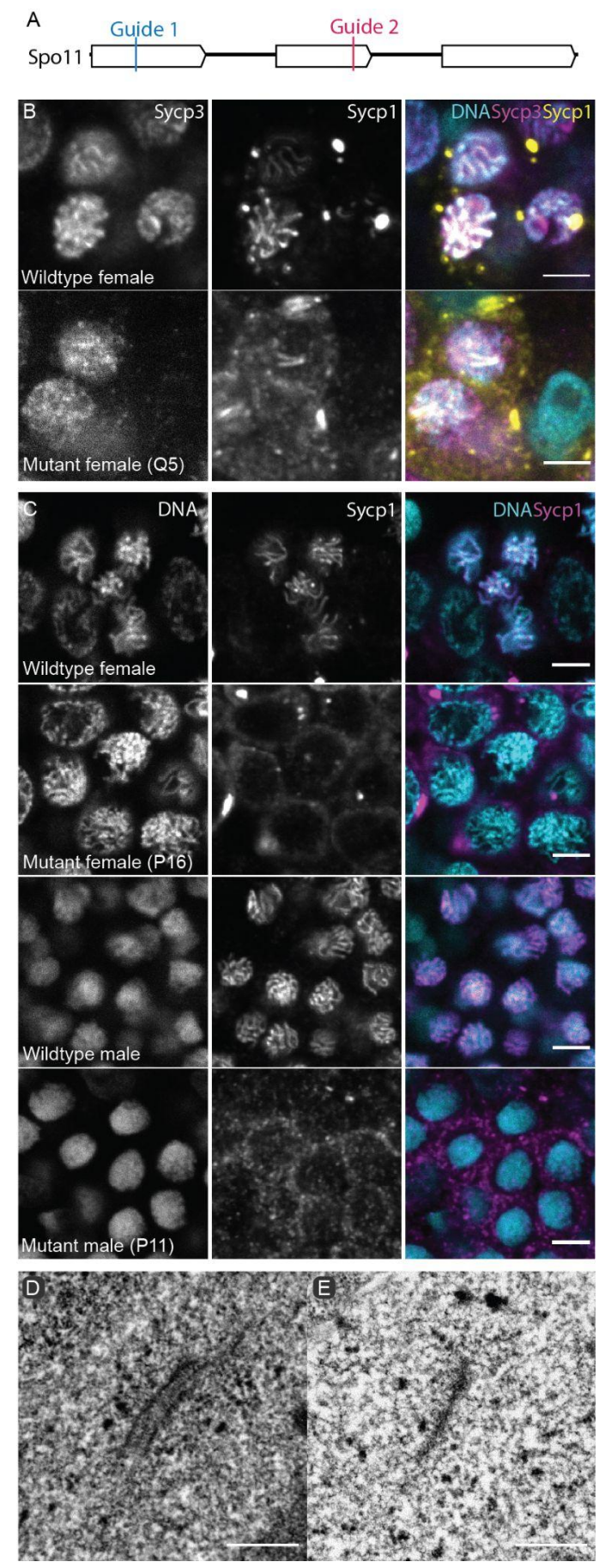

Figure 2. Spo11 is required for synapsis in Clytia hemisphaerica males and females. A. Diagram of the Spo11 exons indicating the target sites of CRISPR/Cas9 guides. B. Confocal planes of wildtype and Spo11 mutant pachytene and pachytene-like nuclei stained with anti-Sycp1, anti-Sycp3, and Hoechst. Scale bar $5 \mu \mathrm{m}$. C. Confocal planes of wildtype and Spo11 mutant pachytene and pachytene-like nuclei stained with anti-Sycp1 and Hoechst. Scale bars 5 $\mu \mathrm{m}$. D. Transmission electron microscope (TEM) image of a wildtype synaptonemal complex. Scale bar $0.5 \mu \mathrm{m}$. E. TEM image of a Spo11 mutant (P16) axial element. Scale bar $0.5 \mu \mathrm{m}$. 

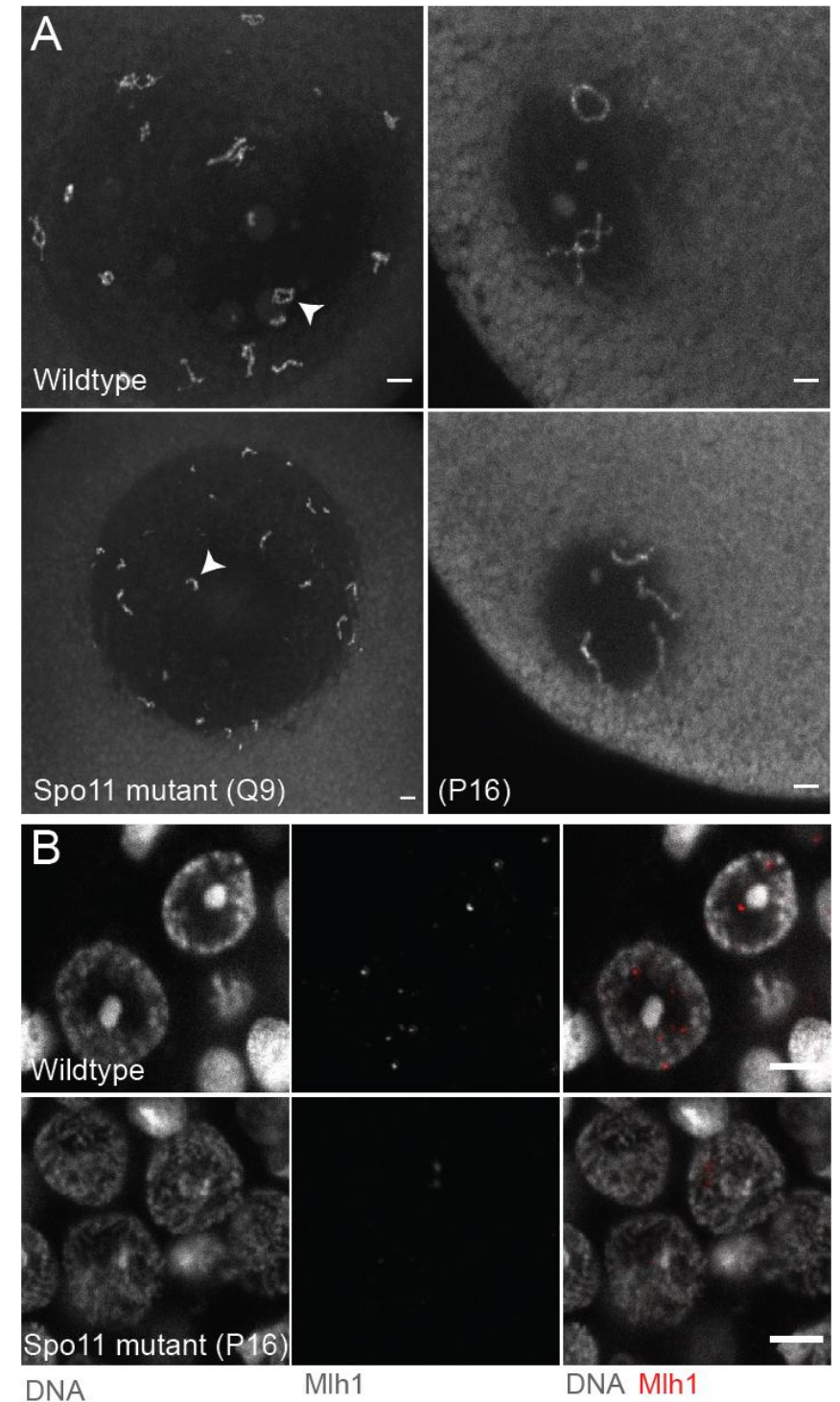

Figure 3. Spo11 is required for crossovers and recombination in Clytia hemisphaerica. A. Fully grown oocytes stained with Hoechst dye. Top left: maximum intensity projection from a confocal z stack of a wildtype oocyte nucleus showing 15 pairs of chromosomes with one or two chiasma per pair. White arrow points to a bivalent. Top right: close up of a wildtype oocyte nucleus showing two homologous chromosome pairs with two chiasma each. Bottom left: maximum intensity projection of a mutant oocyte nucleus showing 30 univalents. White arrow points to a univalent. Bottom right: close up of a mutant oocyte nucleus showing 4 univalents. Scale bar 5 $\mu \mathrm{m}$. B. Wildtype and Spo11 mutant diplotene nuclei stained with anti-Mlh1. Scale bar $5 \mu \mathrm{m}$. 

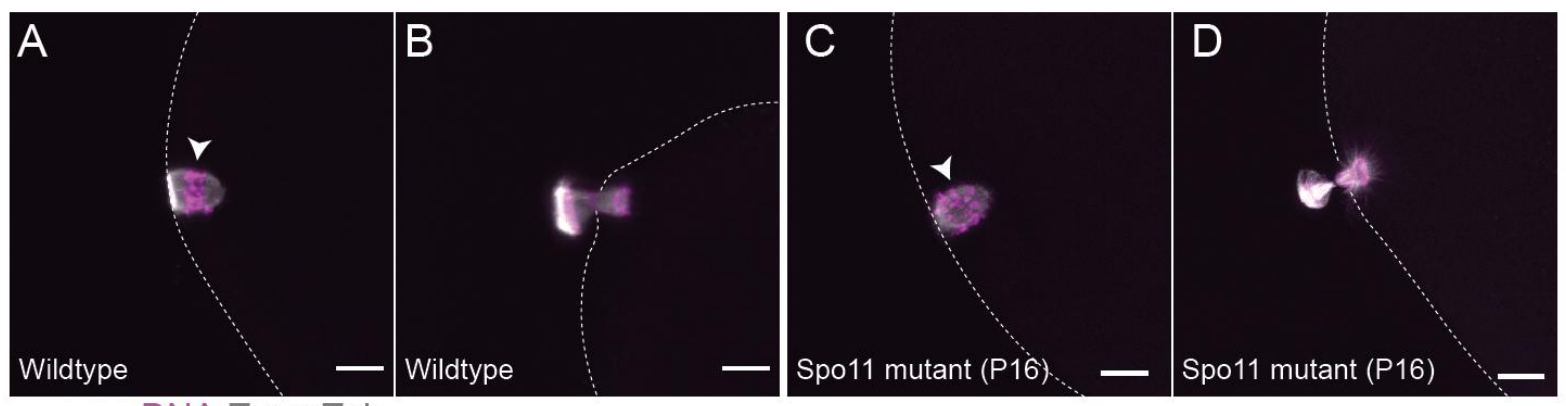

DNA Tyr a-Tub

E

Leptotene Zygotene Pachytene

Fully grown oocyte

Metaphase I

Wildtype
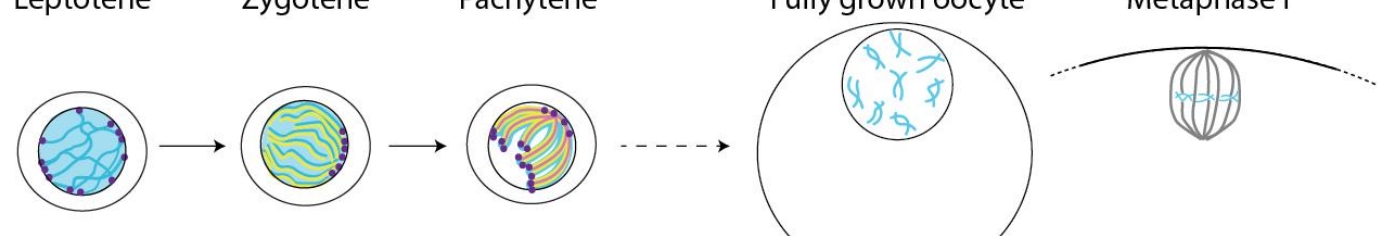

mutant
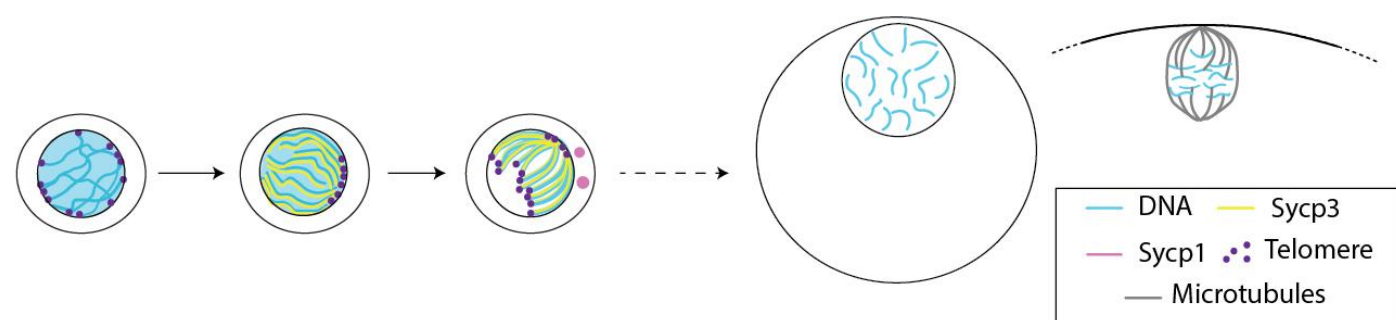

Figure 4. Loss of chiasma results in meiotic division defects in Spo11 mutant oocytes. DNA is stained with Hoechst dye (magenta) while tyrosinated alpha tubulin is stained with anti-tubulin (grey). A. Wildtype metaphase I. White arrow indicates metaphase plate. B. Wildtype during first polar body emission. C. Spo11 mutant metaphase I. White arrow indicates where the metaphase plate should be. D. Spo11 mutant during emission of the first polar body. Scale bar 5 $\mu \mathrm{m}$. E. Summary of meiotic progression from leptotene to the first meiotic division in wildtype and Spo11 mutants. DNA (blue), Sycp3 (yellow), Sycp1 (magenta/pink), telomeres (purple) and microtubules (grey). 